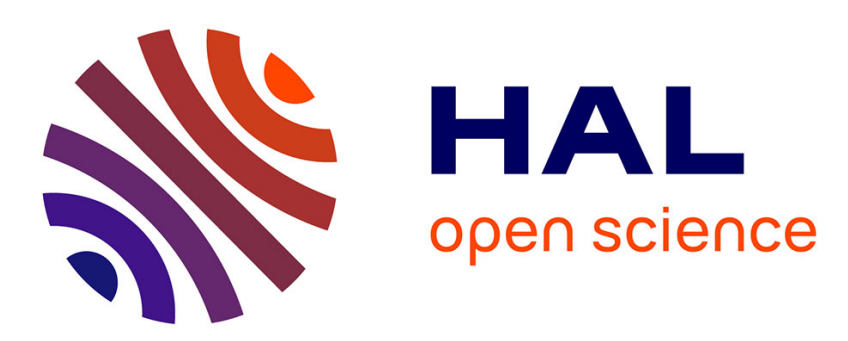

\title{
Implementation of surface tension with wall adhesion effects in a three-dimensional finite element model for fluid flow \\ Michel Bellet
}

\section{- To cite this version:}

Michel Bellet. Implementation of surface tension with wall adhesion effects in a three-dimensional finite element model for fluid flow. Communications in Numerical Methods in Engineering, 2001, 17 (8), pp.Pages 563-579. 10.1002/cnm.430 . hal-00575977

HAL Id: hal-00575977

https://hal-mines-paristech.archives-ouvertes.fr/hal-00575977

Submitted on 12 Mar 2012

HAL is a multi-disciplinary open access archive for the deposit and dissemination of scientific research documents, whether they are published or not. The documents may come from teaching and research institutions in France or abroad, or from public or private research centers.
L'archive ouverte pluridisciplinaire HAL, est destinée au dépôt et à la diffusion de documents scientifiques de niveau recherche, publiés ou non, émanant des établissements d'enseignement et de recherche français ou étrangers, des laboratoires publics ou privés. 


\title{
Implementation of surface tension with wall adhesion effects in a three-dimensional finite element model for fluid flow
}

\author{
Michel Bellet \\ Ecole des Mines de Paris, Centre de Mise en Forme des Matériaux (CEMEF), UMR CNRS 7635, \\ BP 207, F-06904 Sophia Antipolis, France
}

\begin{abstract}
This paper addresses the finite element modelling of surface tension. The external stress vectors associated with surface tension are assumed proportional to the average curvature and oriented along the normal vector to the fluid surface. The issue of estimating the average curvature of a discretized surface is then discussed. It is proposed to use the surface divergence of a field of nodal normal vectors. A special attention is devoted to the calculation so-called consistent normal vectors. In the case of a surface composed of linear triangular facets, it is demonstrated that these normals are given at each node by the average of the normals of the surrounding facets, weighted by their surface. In order to account for wall adhesion effects, the method which consists in modifying the normal vectors at nodes in contact is discussed and extended to the threedimensional case. In order to validate the approach, the three-dimensional finite element resolution of the equilibrium a drop of liquid on a plane is treated.
\end{abstract}

Keywords : surface tension, finite element method, average curvature, consistent normal vectors, wall adhesion 


\section{Introduction}

At the surface of a liquid, surrounded by a gas, some forces develop, due to out of balance molecular forces of attraction acting on the molecules close to the surface [1]. Such molecules are submitted to a resulting force that tends to attract them towards the interior of the fluid, inducing a state of tension in the liquid surface. Such phenomena play an important role in fluid dynamics, as they are able to affect significantly liquid flow through thin tubes or between planes, when surface effects are important. Surface tension effects are also encountered along the surface separating two immiscible fluids.

We consider here a fluid surrounded by an inviscid fluid of pressure $P$. From a macroscopic point of view, surface tension effects reduce to an additional normal stress applied to the fluid surface. Locally, the normal stress vector $\boldsymbol{T}$ will have the following expression, according to the Laplace formula [2]:

$$
\boldsymbol{T}=\boldsymbol{\sigma n}=-P \boldsymbol{n}-\gamma \kappa \boldsymbol{n}
$$

where $\boldsymbol{\sigma}$ is the Cauchy stress tensor, $\boldsymbol{n}$ the outward unit normal vector, $\gamma$ the surface tension coefficient (in units of force per unit length) and $\kappa$ the local average curvature of the surface (assumed positive when the surface is locally convex).

The implementation of such forces in a finite element model depends on the formulation. In the framework of Eulerian formulations, in which the fluid surface is tracked through a fixed mesh (volume of fluid - VOF - or other methods), this raises two problems. First the accuracy to which the free surface is determined is most of the time insufficient for a correct determination of local normal vectors. Second, the determination of the applied surface loading is difficult. To overcome these difficulties, Brackbill et al. [3] propose to represent the vicinity of the fluid surface as a transition region of finite thickness. In this region, the volume of fluid function $F$ varies continuously from 1 to 0 . The normal vector can then be approached by:

$$
\boldsymbol{n}=-\frac{1}{\|\nabla F\|} \nabla F
$$

The authors demonstrate that surface tension forces can then be reduced to volume forces, eliminating the need of surface reconstruction. They have applied this method to two dimensional fluid flow computations.

In order to eliminate any imprecision issued from free surface and associated normal determination, Navti et al. [4] have preferred an arbitrary Lagrangian-Eulerian (ALE) formulation in which only the fluid domain is enmeshed, yielding an explicit description of the free surfaces. In this case, the stress vector issued from (1) is directly entered as an external stress vector in the weak form of the momentum equation. This method has been applied by the authors to different twodimensional cases.

In the present paper, we will present the implementation of surface tension in a threedimensional finite element model for fluid flow, using an ALE formulation. In a first section, the numerical model will be presented. Then the implementation of surface tension will be detailed in section 2. Two methods will be presented for the computation of the average curvature of faceted three-dimensional surfaces. Then, in part 3, wall adhesion effects will be discussed. Finally, in 
section 4, a validation of the method will be presented through an application to the threedimensional resolution of the static equilibrium of a drop of liquid.

\section{Finite element model}

Before discussing the implementation of surface tensions, it is necessary to present briefly the finite element model which has been used.

\section{$\underline{1.1 \text { Governing equations }}$}

The equations governing the motion of an incompressible Newtonian fluid are the following momentum and incompressibility equations :

$$
\left\{\begin{array}{l}
\rho \frac{d \boldsymbol{v}}{d t}-\mu \nabla^{2} \boldsymbol{v}+\nabla p=\rho \boldsymbol{g} \\
\nabla . \boldsymbol{v}=0
\end{array}\right.
$$

where $\boldsymbol{v}$ denotes the fluid velocity, $\rho$ the specific mass, $\mu$ the dynamic viscosity, $p$ the pressure, $g$ the gravity vector.

\section{$\underline{1.2 \text { Boundary conditions }}$}

$\Omega$ denotes the domain occupied by the fluid at a current time $t$ (boundary $\partial \Omega$ ). The boundary conditions are the following.

- Free surface boundary, $\partial \Omega^{\text {free }}$

A given normal stress vector is applied:

$$
\boldsymbol{\sigma n}=\boldsymbol{T}^{d}
$$

This vector $\boldsymbol{T}^{d}$, colinear to the outward unit vector $\boldsymbol{n}$, results from the pressure of the surrounding fluid (default pressure is the atmospheric pressure) and from surface tension, according to $(1)$.

- Solid wall boundary, $\partial \Omega^{\text {wall }}$

A sticking contact condition can be considered $\left(\boldsymbol{v}=\boldsymbol{v}_{\text {wall }}\right)$. In practice, the near wall region is often affected by steep velocity gradients and the sticking contact condition can require a very fine mesh near walls. An alternative consists in using a wall friction law of Newtonian type in which tangential sliding is permitted, the tangential stress vector $\boldsymbol{T}_{f}$ depending on the tangential velocity of the fluid $\boldsymbol{v}_{t}$, according to the relation, $\alpha$ being a friction coefficient:

$$
\boldsymbol{T}_{f}=-\alpha \mu \boldsymbol{v}_{t}
$$

In addition the non-penetration condition has to be fulfilled:

$$
\left(\boldsymbol{v}-\boldsymbol{v}_{\text {wall }}\right) \cdot \boldsymbol{n}=0
$$

\section{$\underline{1.3 \text { Velocity-pressure formulation }}$}


The weak form of previous equations is given hereunder, where velocity and pressure are the primitive variables for which the problem has to be solved

$$
\begin{cases}\forall \boldsymbol{v}^{*} & \int_{\Omega} \mu\left(\nabla \boldsymbol{v}+\nabla \boldsymbol{v}^{T}\right): \boldsymbol{d}^{*} d V-\int_{\Omega} p \nabla \cdot \boldsymbol{v}^{*} d V-\int_{\partial \Omega} \boldsymbol{T} \cdot \boldsymbol{v}^{*} d S-\int_{\Omega} \rho \boldsymbol{g} \cdot \boldsymbol{v}^{*} d V+\int_{\Omega} \rho \frac{d \boldsymbol{v}}{d t} \cdot \boldsymbol{v}^{*} d V=0 \\ \forall p^{*} & \int_{\Omega} p^{*} \nabla \cdot \boldsymbol{v} d V=0\end{cases}
$$

where $p^{*}$ and $v^{*}$ are any virtual velocity and pressure fields, $\boldsymbol{d}^{*}$ being the strain rate tensor associated with $v^{*}$.

\section{$\underline{1.4 \text { Time discretization }}$}

Given the configuration occupied by the fluid at time $t$, the equations to be solved for $(v, p)^{t}$, velocity and pressure field at time $t$, can be expressed in the following way:

$$
\left\{\begin{array}{l}
\forall \boldsymbol{v}^{*} \quad \int_{\Omega^{t}} \mu\left(\nabla \boldsymbol{v}+\nabla \boldsymbol{v}^{T}\right): \boldsymbol{d}^{*} d V-\int_{\Omega^{t}} p^{t} \nabla \cdot \boldsymbol{v}^{*} d V-\int_{\partial \Omega^{t}} \boldsymbol{T}^{t} \cdot \boldsymbol{v}^{*} d S-\int_{\Omega^{t}} \rho \boldsymbol{g} \cdot \boldsymbol{v}^{*} d V+\int_{\Omega^{t}} \rho \frac{\boldsymbol{v}^{t}-\boldsymbol{v}^{t-\Delta t}}{\Delta t} \cdot \boldsymbol{v}^{*} d V=0 \\
\forall p^{*} \quad \int_{\Omega^{t}} p^{*} \nabla \cdot \boldsymbol{v}^{t} d V=0
\end{array}\right.
$$

The time derivative of the velocity is approached by a Euler backward finite difference scheme:

$$
\frac{d v^{t}}{d t}=\frac{1}{\Delta t}\left(v^{t}-v^{t-\Delta t}\right)
$$

where $v^{t}$ and $v^{t-\Delta t}$ are the velocity of the same fluid particle respectively at time $t$ and $t$ - $\Delta t$. The latter is obtained by an advective transport procedure described later. After resolution, the configuration updating is defined by:

$$
\boldsymbol{x}^{t+\Delta t}=\boldsymbol{x}^{t}+\Delta t \boldsymbol{v}^{t}+\frac{\Delta t^{2}}{2} \frac{d \boldsymbol{v}^{t}}{d t}=\boldsymbol{x}^{t}+\Delta t\left(\frac{3}{2} \boldsymbol{v}^{t}-\frac{1}{2} \boldsymbol{v}^{t-\Delta t}\right)
$$

This unconditionally stable Newmark scheme is second order accurate and is also called Adams-Bashforth scheme (AB2). It is equivalent to assume a constant acceleration over each time step [5].

Regarding the non-penetration condition along solid walls, it is enforced by a penalty technique. The normal stress vector is defined by:

$$
\boldsymbol{T}^{t}=-\chi_{p}\left\langle\left(\frac{3}{2} \boldsymbol{v}^{t}-\frac{1}{2} \boldsymbol{v}^{t-\Delta t}-\boldsymbol{v}_{\text {wall }}^{t}\right) \boldsymbol{n}^{t}-\frac{\delta^{t}}{\Delta t}\right) \boldsymbol{n}^{t}
$$

where $\chi_{p}$ is the penalty constant, $\delta^{t}$ is the algebraic distance to the wall at time $t$. The expression between brackets is taken equal to zero when negative (loss of contact).

\section{$\underline{1.5 \text { Finite element discretization and resolution }}$}


Equations (8) are discretized with tetrahedral P1+/P1 elements [6]. The velocity field is linear continuous, including additional degrees of freedom at the centre of the element (bubble formulation), and the pressure field is linear continuous. After elimination of the internal degrees of freedom, the set of discretized equations can be solved for the nodal velocities and pressures.

\subsection{Arbitrary Lagrangian-Eulerian formulation (ALE)}

The basic principle of the ALE method is to separate clearly the mesh velocity field $\boldsymbol{v}_{m s h}$ from the material velocity field $\boldsymbol{v}$. Hence the ALE method is between the Lagrangian method $\left(\boldsymbol{v}_{m s h}=\boldsymbol{v}\right)$ and the Eulerian one $\left(v_{m s h}=0\right)$.

The computation of $\boldsymbol{v}_{m s h}$ consists in regularizing the position of nodes in order to minimize the deformation of the mesh. This is achieved by an iterative procedure which aims at positioning each node at the centre of gravity of the set of its neighbours. This is done under the constraint of conservation of material flux through the domain surface:

$$
\boldsymbol{v}_{\text {msh }} \cdot \boldsymbol{n}=\boldsymbol{v}_{\text {mat }} . \boldsymbol{n}
$$

where $\boldsymbol{n}$ is the outward unit normal. The nodal normal $\boldsymbol{n}$ is taken equal to the consistent normal, as proposed by Gray [10] and Engelman et al. [11]. Its expression is detailed in appendix. The constraint (12) is enforced by a penalty technique. The condition being written here incrementally, the variable $\boldsymbol{v}_{\text {mat }}$ includes the second order acceleration terms of the updating scheme (10):

$$
v_{\text {mat }}=\frac{3}{2} v^{t}-\frac{1}{2} v^{t-\Delta t}
$$

The velocity field $\boldsymbol{v}_{m s h}$ determines the incremental updating of the configuration, according to the following scheme:

$$
\boldsymbol{x}^{t+\Delta t}=\boldsymbol{x}^{t}+\Delta t \boldsymbol{v}_{m s h}
$$

Knowing the mesh velocity, it is necessary to proceed to the updating of nodal fields. Denoting such a field by $B,(B$ can be the temperature $T$ in the case of a thermo-mechanical coupled computation), this is done by writing

$$
B^{t+\Delta t}=B^{t}+\frac{d_{g} B}{d t} \Delta t
$$

where the derivative of $B$ with respect to the grid can be expressed as follows:

$$
\frac{d_{g} B}{d t}=\frac{d B}{d t}-\left(v_{m a t}-v_{m s h}\right) \cdot \nabla B
$$

Once the evolution equation of $B$ has been solved on the time increment, the total derivative of $B$ is known at each node. After computation of $\boldsymbol{v}$ and $\boldsymbol{v}_{m s}$, the updating of $B$ can be obtained by writing (15-16) at each node and only requires the nodal gradient of $B$. Using an upwind technique, this nodal gradient is computed in the upstream element, which is found according to the advection velocity $\boldsymbol{v}_{m s h}-\boldsymbol{v}_{\text {mat }}[6]$.

In order to express the acceleration terms in the momentum equation, a transport of the material velocity field is necessary. In (8), the velocity $\boldsymbol{v}^{t-\Delta t}$ is the material velocity of the particle at 
the previous time level. Hence, after configuration updating, this requires a pure advective transport of the velocity field. This is achieved by a similar scheme as (15-16), but in which the material derivative is taken equal to zero:

$$
\boldsymbol{v}^{t}\left(x^{t+\Delta t}\right)=\boldsymbol{v}^{t}\left(x^{t}\right)-\left(\nabla \boldsymbol{v}^{t}\left(x^{t}\right)\right)\left(v_{m a t}^{t}\left(x^{t}\right)-v_{m s h}\right) \Delta t
$$

\section{Surface tension}

In the above numerical model, the free surface is explicitly described, as a part of the boundary of the finite element mesh. It is then composed of linear triangular facets. The problem is then to compute the local average curvature of such a discretized three-dimensional surface in order to evaluate the second term of (1).

We describe hereunder two methods for this computation. The first one consists in computing the edge curvature for each edge joining two nodes of the surface triangulation, and in cumulating the associated forces at nodes. The second one consists in computing an average normal vector at each node, and then in expressing the curvature at each integration point of the facets by taking the divergence of the field of normal vectors.

\subsection{Method 1: average curvature based on edge curvature}

Let us consider an edge between two nodes $m$ and $n$ (fig. 1). The elements being linear, one of the principal curvature radius can be considered infinite (zero curvature). The average curvature $\kappa$ reduces then to the curvature in the plane orthogonal to the edge direction. An approximate formula is given by [7]:

$$
\kappa=\frac{1}{R}=\frac{l \delta}{A_{e}+A_{f}}
$$

in which $R$ denotes the average curvature radius, $l$ the edge length, $\delta$ the angle between the two adjacent triangles, $A_{e}$ and $A_{f}$ their respective surface. This expression derives from the following analysis. It is to be noticed that the ratios $A_{e} / l$ and $A_{f} / l$ are proportional to the characteristic lengths $h_{e}$ and $h_{f}$ of the facets in the normal direction to the edge, as we have:

$$
A_{e}=\frac{1}{2} h_{e} l \quad \text { and } \quad A_{f}=\frac{1}{2} h_{f} l
$$

This yields:

$$
\frac{A_{e}+A_{f}}{l}=\frac{1}{2}\left(h_{e}+h_{f}\right)
$$

and denoting $h$ the average of $h_{e}$ and $h_{f}$, we can see that (18) is nothing but:

$$
R=\frac{h}{\delta}
$$

This relation can be derived naturally by considering the discretization in linear segments of length $h$ of an arc of circle of radius $R$ (fig. 2). In the triangle OAB, we have: 


$$
R=\frac{\frac{h}{2}}{\sin \frac{\delta}{2}}
$$

In the case of a small desorientation of the facets (i.e. low curvature), the angle $\delta$ is small and (21) is effectively obtained. The increasing difference between formula (21) and (22) with increasing $\delta$ is given by table 1 . When the curvature increases, we can see that the approximate formula (21) provides underestimated values of $R$, and consequently overestimated curvature and associated surface tension terms. However, the maximum difference for curvature is "only" $37 \%$, for extremely sharp edges. Taking into account that this computation is based on an averaged mesh size $h$ around the edge, (21) and consequently (18) appear to be valid.

\section{Implementation in the mechanical equilibrium equation}

The contribution of surface tension terms in the principle of virtual power (7) is:

$$
-\int_{\partial \Omega^{\text {free }}} \boldsymbol{T} \cdot v^{*} d S
$$

In a discretized form, on an edge of the surface, we have from (1) and (18):

$$
\boldsymbol{T}_{\text {edge }}=\gamma \frac{l \boldsymbol{\delta}}{A_{e}+A_{f}} \boldsymbol{n}_{\text {edge }}
$$

where $\boldsymbol{n}_{\text {edge }}$ is the average normal vector on the edge:

$$
\boldsymbol{n}_{e d g e}=\frac{1}{\left\|\boldsymbol{n}_{e}+\boldsymbol{n}_{f}\right\|}\left(\boldsymbol{n}_{e}+\boldsymbol{n}_{f}\right)
$$

The elementary force exerted on the edge can then be expressed as:

$$
\boldsymbol{f}_{\text {edge }}=S_{\text {edge }} \boldsymbol{T}_{\text {edge }}=\left(A_{e}+A_{f}\right) \boldsymbol{T}_{\text {edge }}=\gamma l \delta \boldsymbol{n}_{\text {edge }}
$$

Half of this force is applied to each of the nodes of the edge. The discretization of (23) then leads to:

$$
-\sum_{n \in \partial \Omega^{f r e e}} \sum_{\begin{array}{c}
\text { edge } \\
\text { around } n
\end{array}} \frac{1}{2} \gamma l_{\text {edge }} \delta_{\text {edge }} \boldsymbol{n}_{\text {edge }} . \boldsymbol{V}^{* n}
$$

and the contribution to the residual attached to the degree of freedom $(n, k)$-spatial component $k$ of node $n$-is given by:

$$
R_{n k}^{\text {surf_tens }}=-\sum_{\substack{\text { edge } \\ \text { around } n}} \frac{1}{2} \gamma l_{\text {edge }} \delta_{\text {edge }} \boldsymbol{n}_{\text {edge }} k
$$

The coefficient $1 / 2$ applies when the node $n$ is surrounded by facets belonging to the free surface $\partial \Omega^{\text {free }}$. It is modified when the node belongs to a symmetry plane. It is then necessary to 
distinguish between the contribution of edges which have images by symmetry with respect to the plane and of edges which belong to the symmetry plane.

2.2 Method 2: average curvature computed as the divergence of nodal normal vectors

As demonstrated by Brackbill et al. [3], the average curvature is given by the surface divergence operator:

$$
\kappa=\nabla_{S} \cdot \boldsymbol{n}
$$

in which the spatial partial derivatives are taken in the local tangential plane to the surface. From this relation, an alternative method has been developed to express surface tension forces applied to a faceted three-dimensional surface. This method is composed of the following steps:

- nodal averaging of normal vectors

- computation of the surface divergence of the field of normal vectors, inside each triangle of the surface

- expression of the surface tension terms in the mechanical equilibrium equation

These steps are detailed hereunder.

\section{Nodal averaging of normal vectors}

Each node of the discretized three-dimensional surface belongs to one or several linear triangles. On each plane facet, the normal is unique. Hence, there are essentially two methods of nodal averaging.

The first one is a simple averaging and can be expressed as:

$$
\boldsymbol{n}^{m}=\frac{1}{\left\|\sum_{e \in \tau(m)} \boldsymbol{n}^{e}\right\|} \sum_{e \in \tau(m)} \boldsymbol{n}^{e}
$$

where $\tau(m)$ denotes the set of triangular facets surrounding node $m$ and $\boldsymbol{n}^{e}$ the normal vector to facet $e$. When the average normal to the free surface is looked for, the set of facets $\tau(m)$ is restricted to the facets of the free surface surrounding the node $m$. If the node $m$ belongs to one or two symmetry planes, the average normal vector $n^{m}$ must be projected onto those planes in order that the final averaged vector belong to those planes.

A second method consists in taking $\boldsymbol{n}^{m}$ as the consistent normal vector already defined in the appendix. The normal is then a weighted average of the normal vectors of the facets surrounding the node. The weighting coefficients are simply the surfaces of the facets (see appendix):

$$
\boldsymbol{n}^{m}=\frac{1}{\left\|\sum_{e \in \tau(m)} \boldsymbol{n}^{e} S^{e}\right\|} \sum_{e \in \tau(m)} \boldsymbol{n}^{e} S^{e}
$$

In the sequel, the consistent normal vectors will be used for the estimation of curvature.

\section{Surface divergence of normal vectors}


Let us consider now a linear triangular facet. The three nodes $(m, n, p)$ have averaged normal vectors $\boldsymbol{n}^{m}, \boldsymbol{n}^{n}$ and $\boldsymbol{n}^{p}$. In the plane of the facet, a local coordinate system $(X, Y)$ is defined, where the unit vector $\boldsymbol{e}_{X}$ is tangential to the edge joining the two first nodes of the facet, and $\boldsymbol{e}_{Y}$ is orthogonal to $\boldsymbol{e}_{X}$ (fig. 3). Denoting $(\xi, \eta)$ the coordinates in the reference triangular element, we have:

$$
\begin{aligned}
\nabla_{S} \cdot \boldsymbol{n} & =\frac{\partial n_{X}}{\partial X}+\frac{\partial n_{Y}}{\partial Y} \\
& =\frac{\partial N_{i}}{\partial X} n_{X}^{i}+\frac{\partial N_{i}}{\partial Y} n_{Y}^{i} \\
& =\left(\frac{\partial N_{i}}{\partial \xi} \frac{\partial \xi}{\partial X}+\frac{\partial N_{i}}{\partial \eta} \frac{\partial \eta}{\partial X}\right) n_{X}^{i}+\left(\frac{\partial N_{i}}{\partial \xi} \frac{\partial \xi}{\partial Y}+\frac{\partial N_{i}}{\partial \eta} \frac{\partial \eta}{\partial Y}\right) n_{Y}^{i}
\end{aligned}
$$

in which $N_{i}$ denotes the interpolation function attached to node $i$. The summation on nodes $i(i=m$, $n, p)$ is implicit, using the convention of summation of repeated indices. The derivatives of interpolation functions are constant in the element, whereas the derivatives of the local coordinates $(\xi, \eta)$ with respect to $(X, Y)$ are obtained by expressing the Jacobian matrix $[\partial X / \partial \xi]$ and its inverse, which are also constant in the element. Finally, the divergence of $\boldsymbol{n}$ is obtained by the following formula:

$$
\nabla_{S} \cdot \boldsymbol{n}=\frac{1}{2 S}\left[Y^{p}\left(n_{X}^{n}-n_{X}^{m}\right)-X^{p}\left(n_{Y}^{n}-n_{Y}^{m}\right)+X^{n}\left(n_{Y}^{p}-n_{Y}^{m}\right)\right]
$$

$S$ denotes the surface of the facet. The facets being linear, $\nabla_{S} . \boldsymbol{n}$ is constant on each of them. It can be easily computed after calculating the coordinates of the nodal normals in the local coordinate system $(X, Y)$ attached to each facet. In each point of the facet of local coordinates $(\xi, \eta)$, the expression of the surface tension resulting force is then:

$$
\boldsymbol{T}(\xi, \eta)=-\gamma\left(\nabla_{S} \cdot \boldsymbol{n}\right) \boldsymbol{n}(\xi, \eta)
$$

When $\nabla_{S} \cdot \boldsymbol{n}$ is positive (respectively negative), the surface is locally convex (resp. concave) and the associated surface tension effort is compressive (resp. tensile).

\section{Surface tension terms in the mechanical equilibrium equation}

It is now possible to give a detailed expression of the discrete form of (23). Given a tetrahedral element $e$ having at least one its faces on the boundary, the contribution of $e$ to the residual attached to the degree of freedom $(n, k)$ can be written in the following way.

$$
\begin{aligned}
R_{n k}^{\text {surf_tens }} & =-\int_{\partial \Omega_{e}^{\text {free }}} \boldsymbol{T}_{k} \cdot N_{n} d S \\
& =\int_{\partial \Omega_{e}^{\text {free }}} \gamma\left(\nabla_{S} \cdot \boldsymbol{n}\right) N_{m} n_{k}^{m} \cdot N_{n} d S
\end{aligned}
$$

\section{Wall adhesion effects}

Wall adhesion is the term used to describe the forces acting on the fluid interface along the line of contact with a solid wall (contour of the free surface). In this region are exerted very complex microscopic phenomena, that result in an equilibrium angle $\theta_{e q}$ which is function of the 
different surface free energies which are characteristic of the different interfaces: $\gamma_{S L}$ (solid-liquid interface), $\gamma_{L G}$ (liquid-gas interface) and $\gamma_{S G}$ (solid-gas interface) (fig. 4). The angle $\theta_{e q}$ characterizes the wetting of the wall by the fluid: wetting for $\theta_{e q}<90^{\circ}$, no wetting for $\theta_{e q}>90^{\circ}$. Hence, this angle is not an intrinsic parameter of the fluid, but depends on the contact system. It can be measured experimentally when the fluid is at rest. As mentioned by Temperley and Trevena [8] or Kothe and Mjolsness [9], it is to be noted that this equilibrium angle depends also on the fluid velocity (modulus and orientation with respect to the interface). In the present work, only the static equilibrium angle will be considered.

Following the approach of Brackbill et al. [3], the system of forces applied to the free surface contours is not modelled. It is preferred to account for the establishment of the equilibrium angle along those contours. This is achieved by introducing additional forces which apply to this region when the actual contact angle $\theta$ is different from the static equilibrium one $\theta_{e q}$. In other words, the average curvature is calculated in a specific way in the neighbourhood of the contour of the free surface, in order to generate adequate forces. We shall detail this point.

At this stage, it can be noticed that the introduction of such forces appears to be impossible when using the formulation based on edge curvature. The reason is that, as already said, the edge curvature only provides curvature in the orthogonal direction to the edge. If we consider the contour of the free surface of a three-dimensional fluid domain, it is difficult to imagine how to implement a consistent set of nodal forces issued from edge curvature: around each node in contact with the wall, the edges belonging to the free surface can have very different directions with respect to the contour line. This is the reason why we have extended to 3D the approach suggested by Brackbill in 2D. If the average curvature is computed by the surface divergence of the averaged normal, and if we apply the vectors resulting from (34), it should be possible to account for wall adhesion through a simple modification of the averaged normal vector at nodes in contact.

If we refer to fig. 5, the normal vector $\boldsymbol{n}$ which is used to compute the surface tension forces is modified at each node belonging to the free surface contour, according to the following relation:

$$
\hat{\boldsymbol{n}}=\cos \theta_{e q} \boldsymbol{n}_{w}+\sin \theta_{e q} \boldsymbol{t}_{w}
$$

In this relation, $\boldsymbol{n}_{w}$ is the outward unit vector to the wall surface. Vector $\boldsymbol{t}_{w}$ is tangent to the wall surface, and given by the projection onto the plane of the initial average normal vector to the free surface $\boldsymbol{n}$. This initial $\boldsymbol{n}$ vector is obtained by (31). For the other nodes of the free surface those which are not in contact - we take $\hat{\boldsymbol{n}}$ equal to $\boldsymbol{n}$, and in each facet of the free surface, the contribution of the surface tension to the applied external stress vector is computed as:

$$
\boldsymbol{T}(\xi, \eta)=-\gamma\left(\nabla_{S} . \hat{\boldsymbol{n}}\right) N_{m}(\xi, \eta) \hat{\boldsymbol{n}}^{m}
$$

Figure 6 schematically explains, in 2D, the effect of this local change of vector $\boldsymbol{n}$ when the actual contact angle $\theta$ is different from $\theta_{e q}$. By changing locally the value of $\nabla_{S} \cdot \boldsymbol{n}$, the system of surface tension forces is modified, providing additional forces that will make the interface evolve towards the equilibrium state.

\section{Example of application: static equilibrium of a drop on a plane wall}

The evolution of a liquid drop, initially of cubic shape, in contact with a plane wall is modelled. Starting from an initial cubic configuration, the liquid flow is computed until the drop shape stabilizes. The liquid is submitted to gravity, viscosity, inertia, surface tension including wall 
adhesion as explained in the previous sections. The contact with the wall is frictionless. The dynamic viscosity of the fluid is $\mu=50 \mathrm{~Pa}$.s and its specific mass is $\rho=1000 \mathrm{~kg} \cdot \mathrm{m}^{-3}$. The surface tension coefficient is $\gamma=2 \mathrm{~N} . \mathrm{m}^{-1}$ and three values of the contact angle $\theta_{e q}$ have been considered: 60 , 90 and 120 degrees. The initial cube is $5 \times 5 \times 5 \mathrm{~mm}$. The initial mesh is composed of 1331 nodes, 5000 tetraheadral elements, with 1200 triangular facets on the boundary. The time step is $0.01 \mathrm{~s}$. The evolution of the cube is presented in fig. 7. We can see that the fluid domain configuration converges towards a stable shape, which is reached after $0.5 \mathrm{~s}$ approximatively.

Knowing $\theta_{e q}$, it is possible to calculate analytically the diameter of the circular contact area and the height of the stable configuration. Such a result provides a good reference solution to evaluate the finite element solution. First, the volume of a truncated sphere, of radius $R$ and making a contact angle $\theta_{e q}$ with the truncation plane, is given by:

$$
V=\pi R^{3}\left[\frac{2}{3}-\cos \theta_{e q}+\frac{1}{3} \cos ^{3} \theta_{e q}\right]
$$

The volume conservation ensures $V=V_{0}$, the given initial volume, which yields $R$, the curvature radius of the equilibrium configuration. From $R$, we can deduce the height of the drop $h$ and $\phi$ the diameter of the circular contact area:

$$
h=R\left(1-\cos \theta_{e q}\right) \quad \phi=2 R \sin \theta_{e q}
$$

The comparison between the exact values of $h$ and $\phi$ and those obtained with the finite element model is given in table 2. The differences are very low. Moreover, it is to be noted that the errors indicated in the table include errors of determination, as the dimensions issued from the finite element model are raw results obtained from position of nodes (no interpolation). Another contribution to the error is the slight change in volume that is observed in the finite element computation (a relative loss of volume of $1.2,0.3$ and $0.3 \%$ in the three cases respectively). The treatment of contact by penalty also contributes to the error, since the fluid is allowed to penetrate the wall very slightly (about $10^{-5} \mathrm{~m}$ ).

Hence, these results validate the implementation of surface tension and wall adhesion.

Let us make a last comment about the evaluation of the average curvature. If we consider a node of the free surface far enough from the contact line, the value obtained by averaging the edge curvature of all the edges converging to this node compare well with the value of $R$ obtained by (38), the error being in the range of the errors indicated in table 2. The same remark can be done for the value of $\nabla_{S} . \boldsymbol{n}$ in the facets of the free surface. This validates the two different ways to compute the average curvature of a surface discretized in linear triangles.

\section{Conclusion}

The implementation of surface tension phenomena in a three-dimensional finite element model has been detailed. Regarding the estimation of the average curvature, two different methods have been presented and discussed. In order to take into account wall adhesion effects, the curvature deduced from the surface divergence of normal vectors has been preferred. The method which consist of a local perturbation of these normal vectors has been presented. It has been tested successfully in the case of the static contact of a drop of liquid on a plane. 


\section{References}

1. M. Nelleon, Mechanics and properties of matter, Heineman, London, 1952.

2. L.D. Landau and E.M. Lifshitz, Fluid Mechanics, Pergamon, New York, 1959.

3. J.U. Brackbill, D.B. Kothe and C. Zemach, A continuum method for modeling surface tension, J. Comput. Physics, 100, 335-354, 1992.

4. S.E. Navti, K. Ravindran, C. Taylor and R.W. Lewis, Finite element modelling of surface tension effects using a Lagrangian-Eulerian kinematic description, Comput. Methods. Appl. Mech. Engrg., 147, 41-60, 1997.

5. L. Gaston, A. Kamara and M. Bellet, An Arbitrary Lagrangian Eulerian Finite Element Approach to Non Steady State Turbulent Fluid Flow with Application to Mould Filling in Casting, accepted in Int. J. Num. Meth. Fluids, to be published.

6. M. Bellet and O. Jaouen, Finite element approach of thermomechanics of solidification processes, Proc. Int. Conf. On Cutting Edge of Computer Simulation of Solidification and Casting, Osaka (Japan), I. Ohnaka and H. Yasuda (eds.), The Iron and Steel Institute of Japan, 173-190, 1999.

7. O. Jaouen, Calcul de surfaces minimales discrètes par minimisation de l'intégrale d'énergie (Calculation of discrete minimal surfaces by minimization of energy integral), DEA Report (in french), University of Lyon I, 1995.

8. H.N.V. Temperley and D.H. Trevena, Liquids and their properties. A molecular and macroscopic treatise with application, Ellis Horwood, London, 1978.

9. D.B. Kothe and R.C.Mjolsness, RIPPLE: a new model for incompressible flows with free surfaces, AIAA Journal, 30, 2694-2700, 1992.

10. W.G. Gray, An efficient finite element scheme for two-dimensional surface water computation, Finite Elements in Water Resources, 4-33, 1977.

11. M.S. Engelman, R.L. Sani and P.M. Gresho, The implementation of normal and/or tangential boundary conditions in finite element code for incompressible fluid flow, Int. J. Num. Meth. Engng, 2, 225-238, 1982. 


\section{Appendix: Definition of consistent normal vectors}

The objective of this appendix is to define a set of proper nodal normal vectors, starting from the discretized form of the incompressibility condition. We use then the same reasoning as Gray [10] and Engelman et al. [11], but extended in 3D. In the case of linear spatial interpolation, this will allow us to give a precise expression of consistent nodal normal vectors.

Given $\Omega=\bigcup \Omega_{e}$ the finite element discretization of a computational domain, we have, from the incompressibility equation $(3 b)$ :

$$
\int_{\Omega} \nabla \cdot v d V=0
$$

Equation (A1) is a global form of the incompressibility equation. It corresponds to (7b) in which the particular case $p^{*}=1$ is considered (the interpolation space for pressure must contain the constant functions). The injection of the spatial interpolation of the velocity field $v$ in (A1) yields:

$$
\int_{\Omega} \frac{\partial N_{m}}{\partial x_{i}} V_{i}^{m} d V=V_{i}^{m} \int_{\Omega} \frac{\partial N_{m}}{\partial x_{i}} d V=0
$$

where $N_{m}$ denotes the interpolation function attached to node $m$, and $V_{i}^{m}$ is the component $i$ of the nodal velocity at node $m(i=1,3)$. Using the gradient theorem, we obtain:

$$
V_{i}^{m} \int_{\partial \Omega} N_{m} n_{i} d S=0
$$

The summation in (A3) is then restricted to the nodes $m$ belonging to the boundary $\partial \Omega$ of $\Omega$.The integrals of (A2) and (A3) are in fact computed by summation of integrals on the elements $\Omega_{e}$ the node $m$ belongs to. Then (A3) can be cast under the form:

$$
\sum_{\substack{\text { boundary } \\ \text { nodes } \mathrm{m}}} \boldsymbol{V}^{m} \cdot \boldsymbol{A}^{m}=0
$$

Following the reasoning of Engelman et al., the consistent normal vectors should be such that any tangential nodal velocity (i.e. a velocity which is orthogonal to the consistent normal vector) should not contribute to the external flux (A1). Therefore, we should have:

$$
\text { for any boundary node } m, \quad \boldsymbol{V}_{t g}^{m} \cdot \boldsymbol{A}^{m}=0
$$

where $\boldsymbol{V}_{t g}^{m}$ is the tangential velocity vector at node $m$. This means that for each boundary node $m$, the consistent nodal normal vector $\widetilde{\boldsymbol{n}}^{m}$ should be colinear to $\boldsymbol{A}^{m}$. Its expression is then given by:

$$
\begin{gathered}
\qquad \widetilde{\boldsymbol{n}}^{m}=\frac{1}{\left\|\boldsymbol{A}^{m}\right\|} \boldsymbol{A}^{m} \\
\text { with } A_{i}^{m}=\int_{\partial \Omega} N_{m} n_{i} d S
\end{gathered}
$$


In the specific case of a linear discretization, (A7) reduces to:

$$
A_{i}^{m}=\sum_{e \in \tau(m)} \frac{1}{3} n_{i}^{e} S^{e}
$$

where $\tau(m)$ denotes the set of triangular facets surrounding node $m, \boldsymbol{n}^{e}$ is the normal vector to facet $e$ and $1 / 3$ is the value of the interpolation function attached to the node $m$ at the centre of each surrounding facet. Finally, the consistent normal vector at node $m$ is the average of the normals of the surrounding facets weighted by their surface:

$$
\tilde{\boldsymbol{n}}^{m}=\frac{1}{\left\|\sum_{e \in \tau(m)} \boldsymbol{n}^{e} S^{e}\right\|} \sum_{e \in \tau(m)} \boldsymbol{n}^{e} S^{e}
$$




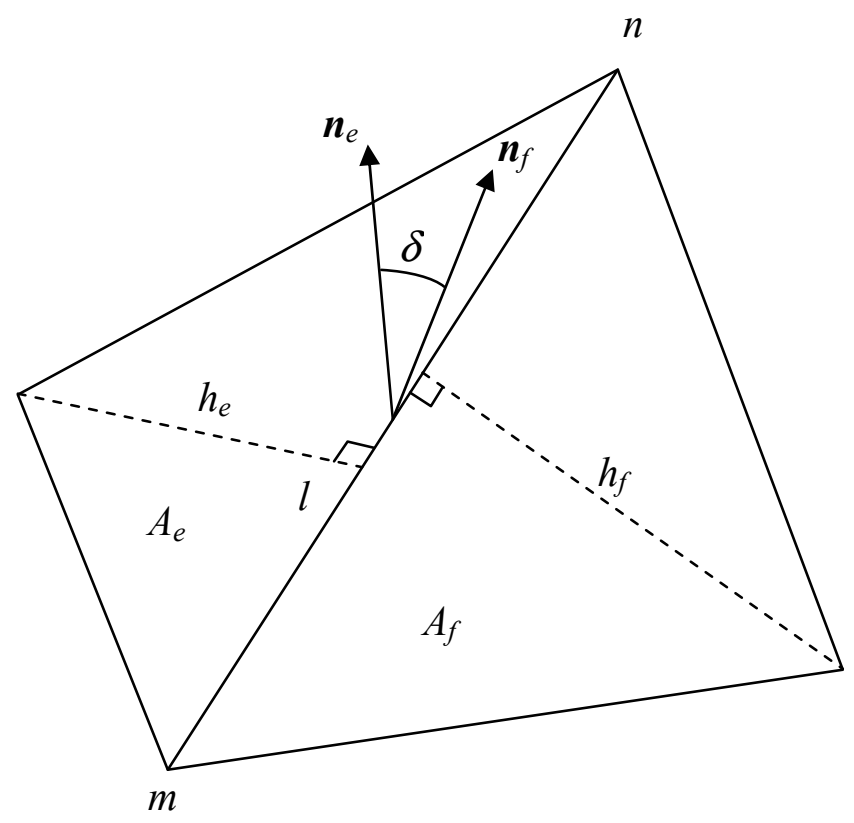

Fig. 1: Notations used in the definition of the curvature along an edge joining two nodes $m$ and $n$ and separating two triangles $e$ and $f$. 


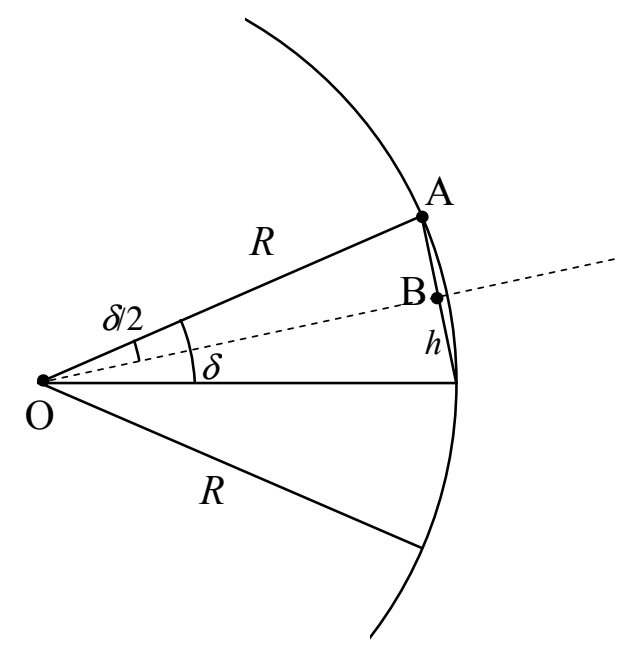

Fig. 2: Arc of circle discretized in linear segments. 


\begin{tabular}{ccc}
$\delta$ (degrees) & $R=(h / 2) / \sin (\delta / 2)$ & $R=h / \delta$ \\
\hline 1 & 57.30 & 57.30 \\
10 & 5.74 & 5.73 \\
60 & 1.93 & 1.91 \\
90 & 1.00 & 0.95 \\
120 & 0.71 & 0.64 \\
150 & 0.58 & 0.48 \\
\end{tabular}

Table 1. Comparison of exact and approximate formulae for the calculation of the radius of curvature from the edge curvature. 

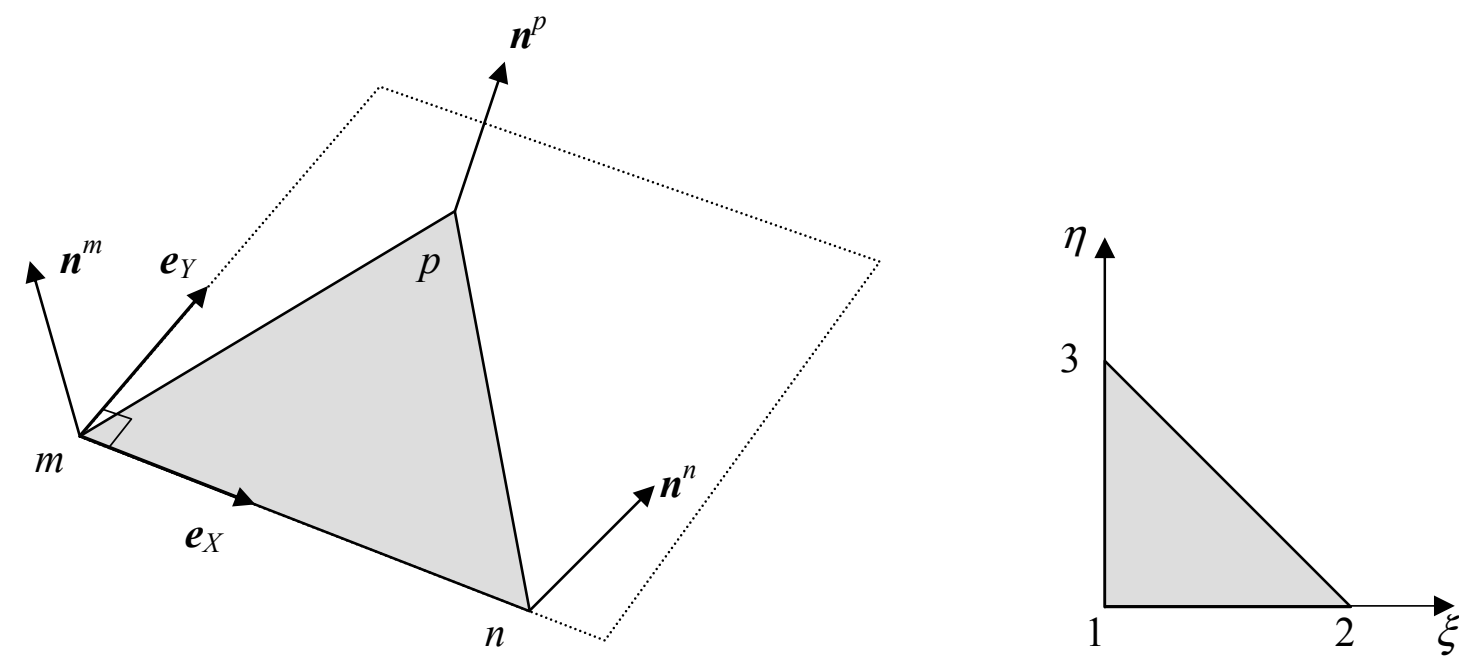

Fig. 3: Local system of coordinates used to compute $\nabla_{S} . \boldsymbol{n}$ in each triangular facet. 


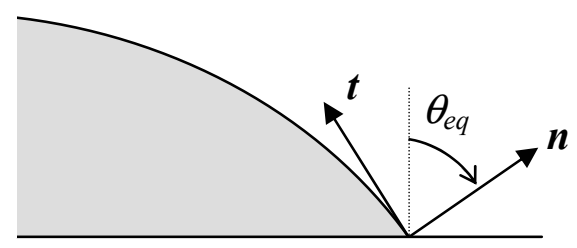

wetting of the wall
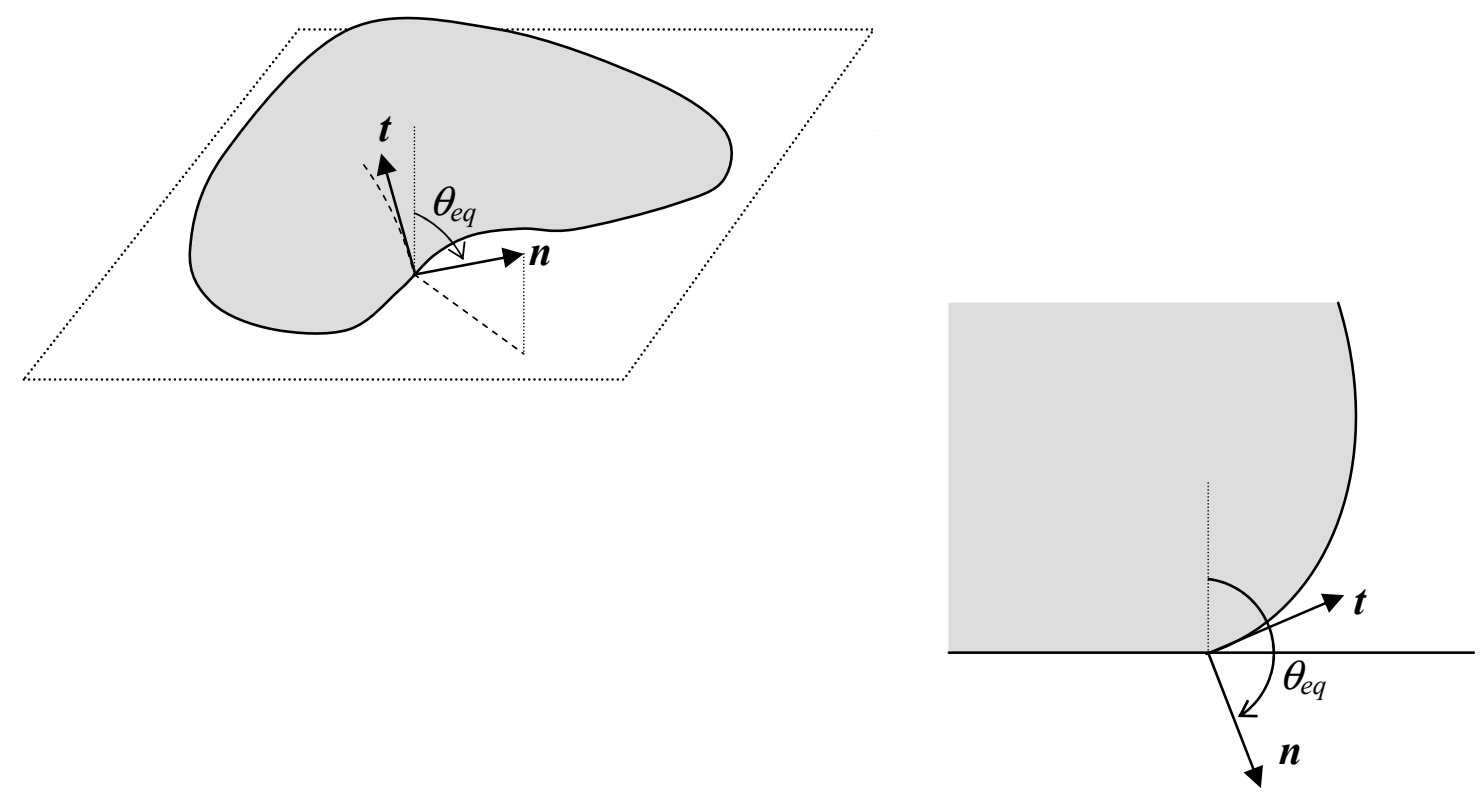

no wetting of the wall

Fig. 4: Wall adhesion. On the left, three-dimensional representation. On the right, two-dimensional representation in the case of wetting and no wetting conditions. 


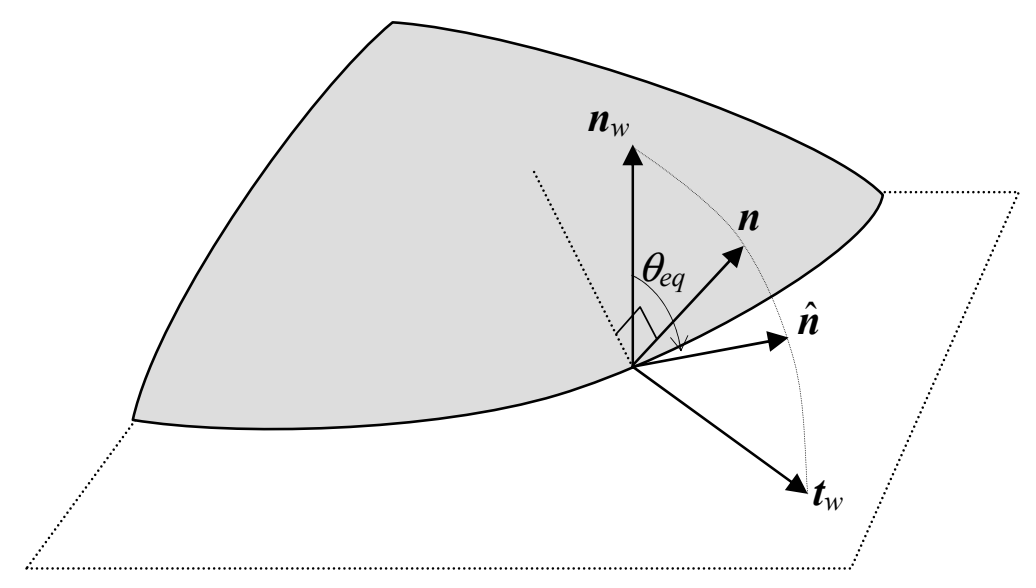

Fig. 5: Modification of the normal vector for a node belonging to the contour of the free surface. 


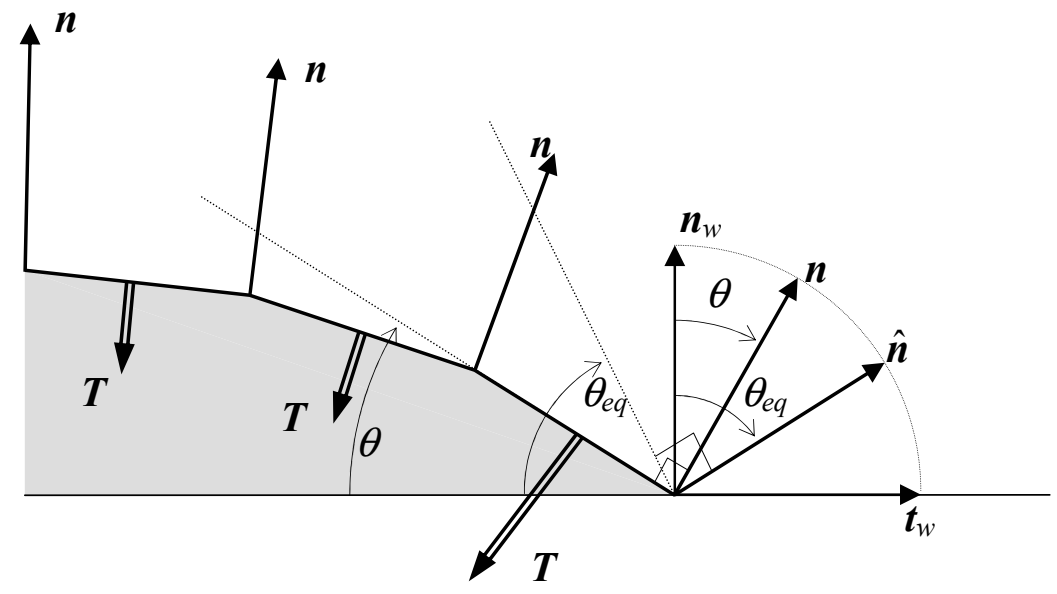

Wetting and $\theta<\theta_{e q}$ : stresses $\boldsymbol{T}$ are locally increased.

They tend to increase $\theta$.

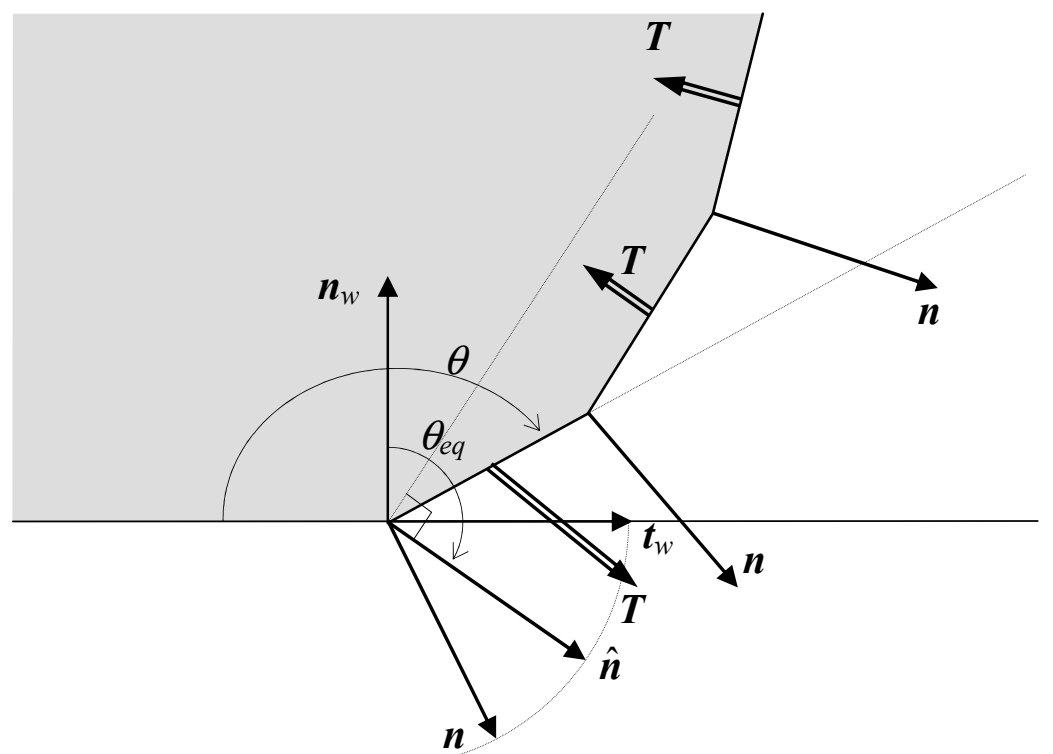

Non wetting and $\theta>\theta_{e q}$ : stresses $\boldsymbol{T}$ are locally increased and their sign is changed. They tend to decrease $\theta$.

Fig. 6: Schematic explanation of the effect of the additional stresses due to the change of $\boldsymbol{n}$. 

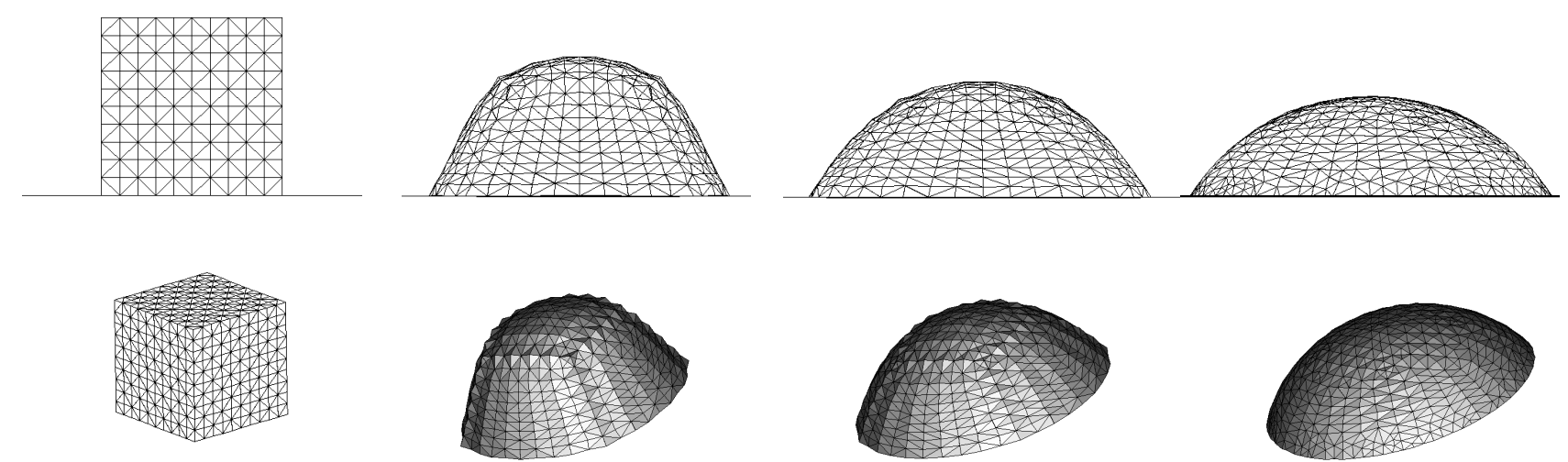

$$
\theta_{e q}=60^{\circ}
$$
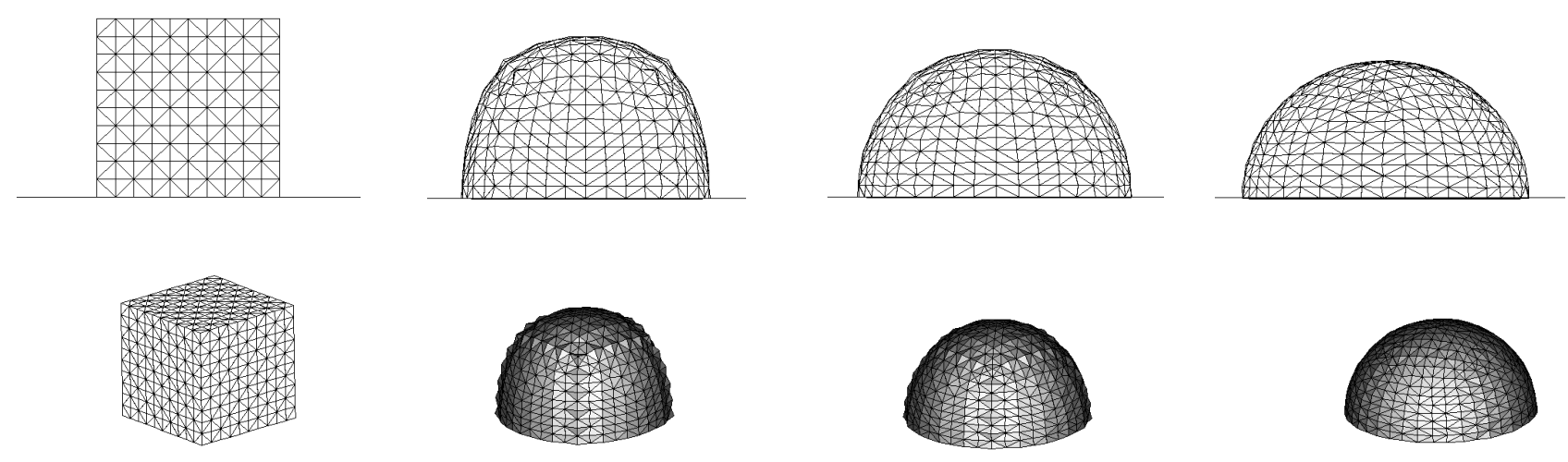

$$
\theta_{e q}=90^{\circ}
$$
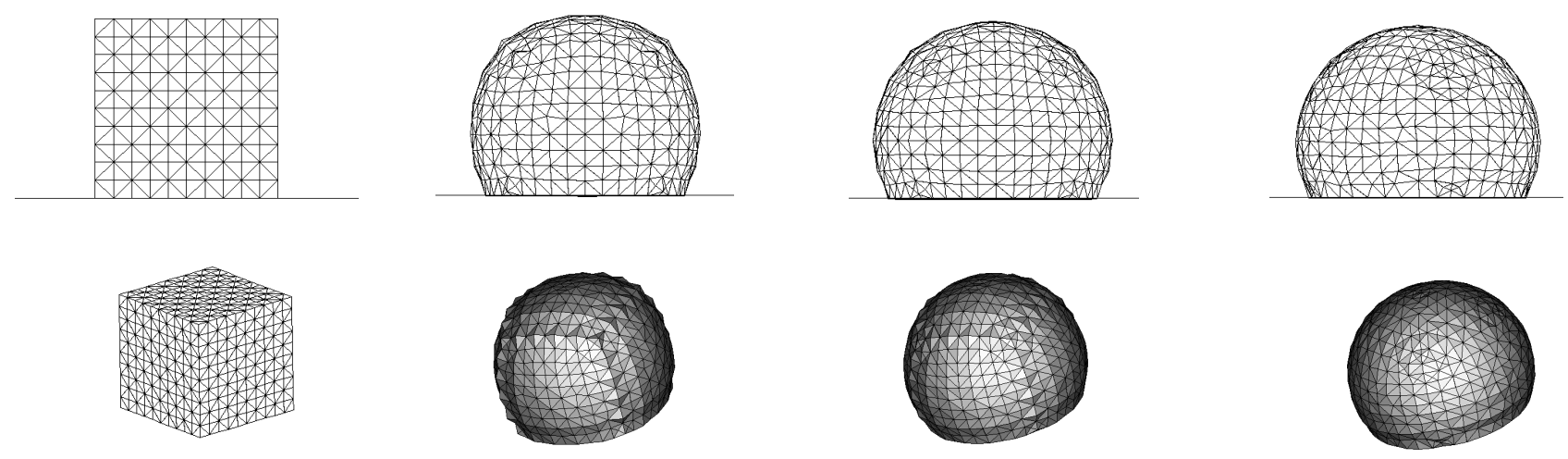

$$
\theta_{e q}=120^{\circ}
$$

Fig. 7: Finite element computation of the evolution of a volume of liquid, initially cubic, towards its equilibrium state, for three different contact angles. In each case, profile view on the first line and perspective on the second line. 


\begin{tabular}{cccccccc}
\hline \multirow{2}{*}{ Contact angle $\theta_{e q}$} & \multicolumn{3}{c}{ Base diameter of the drop } & \multicolumn{3}{c}{ Height of the drop } \\
& $\phi_{e x}(\mathrm{~mm})$ & $\phi(\mathrm{mm})$ & Error $(\%)$ & $h_{e x}(\mathrm{~mm})$ & $h(\mathrm{~mm})$ & Error (\%) \\
\hline 0 & 9.975 & 9.966 & -0.09 & 2.879 & 2.828 & -1.78 \\
90 & 7.816 & 7.870 & 0.69 & 3.908 & 3.830 & -2.00 \\
120 & 5.685 & 5.928 & 4.3 & 4.923 & 4.810 & -2.3 \\
\hline
\end{tabular}

Table 2: Static equilibrium of a liquid drop on a plane wall. Comparison between the drop characteristic dimensions obtained by analytical solution and finite element model. 